\title{
Analysis of optimal control models for the human locomotion
}

\author{
Yacine Chitour, Francesca Chittaro, Frédéric Jean and Paolo Mason
}

\begin{abstract}
In recent papers it has been suggested that human locomotion may be modeled as an inverse optimal control problem. In this paradigm, the trajectories are assumed to be solutions of an optimal control problem that has to be determined. We discuss the modeling of both the dynamical system and the cost to be minimized, and we analyse the corresponding optimal synthesis. The main results describe the asymptotic behavior of the optimal trajectories as the target point goes to infinity.
\end{abstract}

\section{INTRODUCTION}

In the recent papers [2], [3] it has been conjectured that human locomotion is governed by optimality criteria. Consider for instance a person walking in a empty room, whose purpose is to walk from the actual position to another position, reaching a prescribed final direction. Intuitively one could conjecture that the path chosen by the subject will not be far from the straight line, i.e. the shortest and fastest path. However, in general, if the velocity is never vanishing and the dynamics is assumed to be nonholonomic, as suggested for instance by the experimental results of [1], the straight line turns out to be unfeasible and the minimum time problem is not well defined.

The idea of [2], [3] is that the choice of the path also depends on the "effort" made in order to modify the direction of the motion. More precisely the chosen trajectory is assumed to minimize a compromise between the length of the path (or the time needed to reach the final point) and an energy term, which will depend on the curvature $\kappa$ or its derivatives. The experimental paths measured and discussed in [2] suggest in particular a major role of the variation of the curvature in the latter energy term.

The purpose of this paper is to propose and study a rather general family of optimal control problems whose solutions are candidates to model the trajectories spontaneously chosen in human locomotion, in the spirit of [2], [3]. By a qualitative analysis of these optimal control problems we are able to detect an asymptotic behaviour of the shape of the corresponding optimal trajectories, in case the initial point and the target are far enough. This behavior is qualitatively compatible with the paths spontaneously chosen during human locomotion. Therefore, in principle, a numerical study of the optimal trajectories and a comparison with the trajectories experimentally recorded (as in [2]) could determine which is

This paper was supported by the Digiteo grant Congeo.

Y. Chitour, F. Chittaro and P. Mason are with Laboratoire des signaux et systèmes, Université Paris-Sud, CNRS, Supélec, 91192 Gif-Sur-Yvette, France, yacine.chitourdss.supelec.fr, paolo.masonelss.supelec.fr.

F. Jean is with École Nationale Supérieure de Techniques Avancées, 75739 Paris cedex 15 France, Frederic. Jean@ensta.fr. the optimal control problem among those considered which best fits the experimental data. In this paper we will actually not be concerned with this inverse optimal control problem.

The paper is organized as follows. In Section II we introduce the notations used throughout the paper and we define the family of optimal control problems that we study. In Section III we state the main results of the paper. In particular we first prove the existence of an optimal trajectory satisfying the PMP in Sections III-A and III-B. We then determine some useful a priori estimates on the cost in Section III-C and some important qualitative properties of the optimal trajectories in Section III-D. In Section IIIE we complete the qualitative analysis and we suggest a numerical method to compute optimal trajectories for far enough targets. Finally, in Section IV we discuss the possible future research directions. Note that, for reasons of space, we will essentially not provide the proofs of our results, which will be reserved to a forthcoming publication.

\section{NOTATIONS AND DEFINITION OF THE OPTIMAL CONTROL PROBLEM}

We start this section by introducing the notations that will be used throughout the paper.

We will always assume without loss of generality that the difference $\alpha_{1}-\alpha_{2}$ between two angles $\alpha_{1}, \alpha_{2} \in[0,2 \pi]$ takes values in the interval $[-\pi, \pi]$. In particular with this notation the modulus $\left|\alpha_{1}-\alpha_{2}\right|$ is a continuous function of $\alpha_{1}, \alpha_{2}$ taking values in $[0, \pi]$.

Given a subset $S$ of $[0, T]$ we will denote its Lebesgue measure by $\mu(S)$.

The scalar product in $\mathbb{R}^{2}$ is denoted by $\langle\cdot, \cdot\rangle$.

The symbol $B(x, r)$ indicates the ball of radius $r$ centered at $x$.

As usual, given two subsets $A, B$ of a vector space the set $A+B$ is defined as

$$
A+B=\{x+y: x \in A, y \in B\} .
$$

We now define the family of optimal control problems that will be studied throughout the paper. In order to simplify the setting we assume that these optimal control problems are associated with the following common dynamics

$$
\left\{\begin{array}{l}
\dot{x}=\cos \theta, \\
\dot{y}=\sin \theta, \\
\dot{\theta}=\kappa, \\
\dot{\kappa}=u,
\end{array}\right.
$$

where $(x, y, \theta, \kappa)$ belongs to $\mathbb{R}^{2} \times S^{1} \times \mathbb{R}$ and $u \in \mathbb{R}$. The previous system describes the non-holonomic dynamics of 
a rather simple object (representing a vehicle or a human being) on the plane detected by the position on the plane and the angle with a prescribed direction, where it is assumed that the velocity is constant (and, up to time rescaling, equal to 1). Actually the last assumption, which is rather strong, turns out to be quite realistic if the starting point and the target are far enough.

The dynamics is controlled through the angular acceleration $u$. In particular we assume that admissible controls are measurable functions defined on an interval $[0, T]$ where $T>0$ depends in general on $u$.

Given arbitrary $X_{0}$ and $X_{1}$ in $\mathbb{R}^{2} \times \mathcal{S}^{1} \times \mathbb{R}$, the optimal control problem is then defined by the following cost

$$
J(u(\cdot), T)=\int_{0}^{T}[1+\varphi(\kappa(t))+\psi(u(t))] d t,
$$

which should be minimized among all trajectories of the system steering $X_{0}$ to $X_{1}$. Here the functions $\varphi$ and $\psi$ verify the following hypotheses

(H1) $\varphi$ and $\psi$ are non negative, $\mathcal{C}^{2}$ and even functions defined on $\mathbb{R}$, and are non decreasing on $\mathbb{R}^{+}$. Moreover, $\varphi(0)=$ $\psi(0)=0$

(H2) $\psi$ is strictly convex and $\psi^{\prime \prime}(0)>0$;

(H3) there exist $p>1$ and two positive constants $C, R$ such that

$$
\psi(r) \geq C|r|^{p}, \text { for every } r \in \mathbb{R} \text { such that }|r| \geq R .
$$

The fact that $\varphi$ and $\psi$ are assumed to be even is motivated by trivial symmetries of the problem but is not technically relevant. The other assumptions are classical and crucial in order to perform the qualitative analysis which follows. Notice that the cost defined above is a compromise between the total time $T$ (equivalently, the length to be covered) and an "energy term" depending separately on the curvature $\kappa$ and its variation $u$.

Since the only relevant coordinates for the subject at rest are the spatial and angular components, two reasonable conditions could be assumed at the initial and final points $X_{0}, X_{1}$. On one hand it is possible to look for the trajectory minimizing $J(u(\cdot), T)$ among all the points $X_{0}, X_{1}$ with fixed spatial and angular components, letting the curvature free. On the other hand one could impose the condition $\kappa=0$ at $X_{0}, X_{1}$. This second hypothesis has been considered in [2] because of the particular experimental setting. In accordance with previous literature, in this paper we will follow this assumption, though the alternative assumption essentially leads to the same results.

Our optimal control problem can then be summarized as follows.

(OCP) Fix an initial point $X_{0}=(0,0, \pi / 2,0)$. For every final point of the form $X_{1}=\left(x_{1}, y_{1}, \theta_{1}, 0\right)$, for some $\left(x_{1}, y_{1}, \theta_{1}\right) \in \mathbb{R}^{2} \times S^{1}$, find the trajectories of (1) steering the system from $X_{0}$ to $X_{1}$ and minimizing $J(u(\cdot), T)$.

\section{QUALITATIVE ANALYSIS OF THE OPTIMAL TRAJECTORIES}

In this section we analyse and detect some important qualitative properties of the solutions of the optimal control problems defined in the previous section. Note that similar qualitative properties have been obtained in [5], [6] for a very specific cost.

\section{A. Existence of optimal trajectories}

Note that, by using classical tools, it is easy to see that the control system (1) is controllable (see for instance [5]).

The existence of solutions to problem (OCP) is then guaranteed by the following result, which is obtained by rather classical methods and is related to the weak $L^{p}$ compactness of the minimizing sequences under the given assumptions on $\varphi, \psi$.

Proposition 3.1: For every choice of $X_{0}$ and $X_{1}$ in $\mathbb{R}^{2} \times$ $\mathcal{S}^{1} \times \mathbb{R}$ there exists a trajectory $\bar{X}(\cdot)$ of $(1)$, defined on $[0, \bar{T}]$, associated to some control $\bar{u}(\cdot)$ and minimizing $J(u(\cdot), T)$ among all the trajectories starting from $X_{0}$ and reaching $X_{1}$.

\section{B. Application of the Pontryagin maximum principle}

In order to apply the PMP, one usually needs to know that the optimal control $\bar{u}(\cdot)$ is bounded in the $L^{\infty}$ topology. At the present stage of the analysis, we do not possess that information and we therefore must rely on more sophisticated versions of the PMP. For instance, one readily checks that (OCP) meets all the hypotheses required in Theorem 2.3 of [4] and we get the following.

Proposition 3.2: Let $\bar{X}(\cdot)$ be an optimal trajectory for (OCP), defined on $[0, \bar{T}]$ and associated to the control $\bar{u}(\cdot)$. Then this trajectory satisfies the PMP.

The Hamiltonian of system (1) is:

$$
\begin{aligned}
H= & H(X, p, u, \nu) \\
= & p_{1} \cos \theta+p_{2} \sin \theta+p_{3} \kappa \\
& +p_{4} u-\nu(1+\varphi(\kappa)+\psi(u)),
\end{aligned}
$$

where $p=\left(p_{1}, p_{2}, p_{3}, p_{4}\right) \in \mathbb{R}^{4}$ is the adjoint vector and $\nu \geq 0$.

The PMP writes as follows. Let $u(\cdot)$ be an optimal control defined on the interval $[0, T]$ and $X(\cdot)$ the corresponding optimal trajectory. (By the result of the previous paragraph, such a control exists.) Then $X(\cdot)$ is an extremal trajectory, i.e. it satisfies the following conditions. There exists an absolutely continuous function $p:[0, T] \rightarrow \mathbb{R}^{4}$ and $\nu \leq 0$ such that the pair $(p(\cdot), \nu)$ is non-trivial, and such that we have:

$$
\left\{\begin{array}{l}
\dot{X}(t)=\frac{\partial H}{\partial p}(X(t), p(t), \nu, u(t)), \\
\dot{p}(t)=-\frac{\partial H}{\partial X}(X(t), p(t), \nu, u(t)) .
\end{array}\right.
$$

As $(X(0), X(T))$ is fixed, there is no transversality condition on $(p(0), p(T))$, and the system being autonomous, the Hamiltonian is conserved along extremal trajectories. The maximization condition writes:

$$
\begin{aligned}
& H(X(t), p(t), u(t), \nu) \\
& \quad=\max _{v \in \mathbb{R}} H(X(t), p(t), v, \nu), \quad \forall t \in[0, T] .
\end{aligned}
$$


As the final time is free, the Hamiltonian is zero (see [8]):

$$
H(X(t), p(t), u(t), \nu)=0, \quad \forall t \in[0, T] .
$$

The equation on the covector $p$, also called adjoint equation, becomes:

$$
\left\{\begin{array}{l}
\dot{p}_{1}=0, \\
\dot{p}_{2}=0, \\
\dot{p}_{3}=p_{1} \sin \theta-p_{2} \cos \theta, \\
\dot{p}_{4}=-p_{3}+\nu \varphi^{\prime}(\kappa) .
\end{array}\right.
$$

If $\nu \neq 0$ we can always suppose, by linearity of the adjoint equation, that $\nu=1$. In this case (resp., if $\nu=0$ ) a solution of the PMP is called a normal extremal (resp., an abnormal extremal). It is easy to see that all optimal trajectories are normal extremals. Indeed, if $\nu=0$, then $p_{4} \equiv 0$ by the maximization condition (6). From $\dot{p}_{4}=0$, we immediately deduce that $p_{3} \equiv 0$ and, from $\dot{p}_{3}=0$, it turns out that $p_{1} \sin \theta-p_{2} \cos \theta \equiv 0$. From $H=0$, one also has $p_{1} \cos \theta+$ $p_{2} \sin \theta$ and thus $p_{1}=p_{2}=0$. That contradicts the nontriviality of $(p, \nu)$.

Consequently, equation (7) becomes

$$
p_{1} \cos \theta+p_{2} \sin \theta+p_{3} \kappa+p_{4} u-(1+\varphi(\kappa)+\psi(u))=0 .
$$

As regards the maximization condition (6), the optimal control is given by

$$
u(t)=\left(\psi^{\prime}\right)^{-1}\left(p_{4}(t)\right) \quad \text { for } t \in[0, T] .
$$

Note that the strict convexity and the growth condition on $\psi$ imply that $\psi^{\prime}$ realizes a bijection from $\mathbb{R}$ to $\mathbb{R}$ and thus its inverse is a continuous and strictly increasing function from $\mathbb{R}$ to $\mathbb{R}$.

From (8), we get that $p_{1}$ and $p_{2}$ are constant. Therefore from the Hamiltonian system (5) we get that, along an optimal trajectory, the following equation, independent of $u$, is satisfied on $[0, T]$ and for a suitable choice of $\left(p_{1}, p_{2}\right) \in$ $\mathbb{R}^{2}$,

$$
\left\{\begin{array}{l}
\dot{\theta}=\kappa, \\
\dot{\kappa}=\left(\psi^{\prime}\right)^{-1}\left(p_{4}\right), \\
\dot{p}_{3}=p_{1} \sin \theta-p_{2} \cos \theta, \\
\dot{p}_{4}=-p_{3}+\varphi^{\prime}(\kappa) .
\end{array}\right.
$$

\section{A uniform estimate of the cost}

We next provide a proposition which allows to estimate the cost of an optimal trajectory.

Proposition 3.3: Given $\sigma>0$, a pair $\left(\lambda_{0}, \lambda_{1}\right) \in \mathbb{R}_{+}^{2}$ and $T \geq 2 \lambda_{0}+2 \lambda_{1}$ we define the control function

$$
u(t)=\left\{\begin{array}{rl}
-\sigma & t \in\left[0, \lambda_{0}\right] \\
+\sigma & t \in\left(\lambda_{0}, 2 \lambda_{0}\right] \\
0 & t \in\left(2 \lambda_{0}, T-2 \lambda_{1}\right] \\
+\sigma & t \in\left(T-2 \lambda_{1}, T-\lambda_{1}\right] \\
-\sigma & t \in\left(T-\lambda_{1}, T\right]
\end{array} .\right.
$$

Then, for every choice of $X_{1}=\left(x_{1}, y_{1}, \theta_{1}, 0\right)$ with $\left|\left(x_{1}, y_{1}\right)\right| \geq 8 \sqrt{\pi / \sigma}$, there exists a pair $\left(\lambda_{0}, \lambda_{1}\right) \in$ $[0, \sqrt{3 \pi / \sigma}] \times[0, \sqrt{5 \pi / \sigma}]$ and $T \geq 2 \lambda_{0}+2 \lambda_{1}$ such that the trajectory of (1) with $u(\cdot)$ given by (12) starting at $X_{0}$ reaches $X_{1}$ at time $T$.

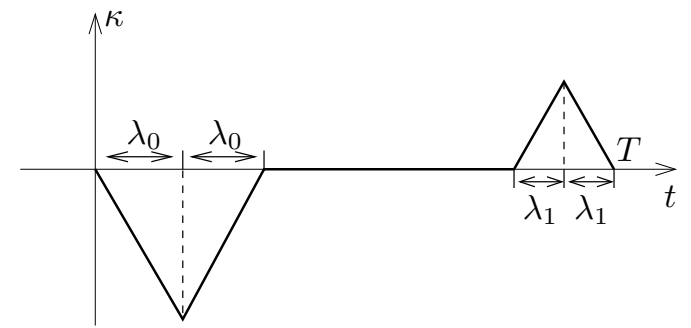

Fig. 1. The function $\kappa(\cdot)$ associated to the control function of Proposition 3.3

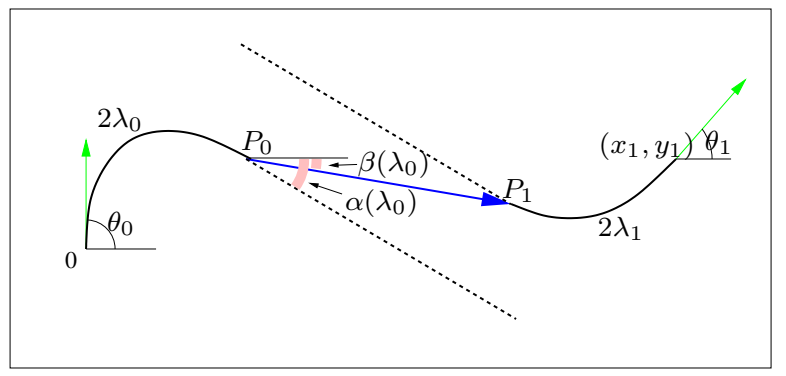

Fig. 2. Proposition 3.3: the reference trajectory corresponds to the situation $\alpha\left(\lambda_{0}\right)=\beta\left(\lambda_{0}\right)$

Comparison with the reference trajectories defined above leads to relevant estimates on optimal trajectories, as shown by the following proposition.

Proposition 3.4: For every $R>0$ there exists a constant $C_{\varphi, \psi}$ depending on $\varphi, \psi$ and $R$ such that the following holds. For every $X_{1}$ such that $\left|\left(x_{1}, y_{1}\right)\right| \geq R$ and if $u(\cdot)$ is an optimal control defined on $[0, T]$ steering the system from $X_{0}$ to $X_{1}$ the following relations hold

$$
\left|\left(x_{1}, y_{1}\right)\right| \leq T \leq J_{\mu}(u(\cdot), T) \leq\left|\left(x_{1}, y_{1}\right)\right|+C_{\varphi, \psi} .
$$

Consequently,

$$
\int_{0}^{T}(\varphi(\kappa(t))+\psi(u(t))) d t \leq C_{\varphi, \psi} .
$$

Remark 3.5: For every $\varepsilon>0$ and every optimal control $u$ defined on $[0, T]$, let $\mathcal{U}_{\varepsilon}$ be the subset of $[0, T]$ given by

$$
\mathcal{U}_{\varepsilon}=\{t \in[0, T]:|u(t)| \geq \varepsilon\} .
$$

From Equation (14) and the strict convexity of $\psi$, we deduce that for every $\varepsilon>0$ there exists a positive constant $\hat{C}_{\varphi, \psi}$ such that for every $u$ defined on $[0, T], \mu\left(\mathcal{U}_{\varepsilon}\right) \leq \hat{C}_{\varphi, \psi}$.

As a consequence of (14) we easily get the uniform equicontinuity of the $\kappa$ components of the optimal trajectories, solutions of (OCP). This is a particular case of the following result.

Proposition 3.6: For every $\Gamma>0$ and $\varepsilon>0$ there exists $\delta_{\varepsilon, \Gamma}>0$ such that $\left|s_{1}-s_{2}\right| \leq \delta_{\varepsilon, \Gamma}$ implies $\left|\kappa\left(s_{1}\right)-\kappa\left(s_{2}\right)\right| \leq$ $\varepsilon$ for every $\left[s_{1}, s_{2}\right] \subset\left[t_{1}, t_{2}\right]$, whenever $t_{1}, t_{2}$ and the optimal control $u(\cdot)$ are such that $\int_{t_{1}}^{t_{2}} \psi(u(s)) d s \leq \Gamma$. Moreover $\lim _{\Gamma \rightarrow 0} \delta_{\varepsilon, \Gamma}=+\infty$.

\section{Qualitative asymptotic results}

In this section we will present some asymptotic results on the structure of optimal trajectories. The first results 
could actually be consider as technical lemmas (together with others, that here we neglect) in order to prove the main asymptotic results. They are stated here since they show rather interesting properties of optimal trajectories.

Let $\alpha \in[0,2 \pi)$ be such that $\left(x_{1}, y_{1}\right)=$ $\left|\left(x_{1}, y_{1}\right)\right|(\cos \alpha, \sin \alpha)$ and let us write as $\left(p_{1}, p_{2}\right)=$ $\rho(\cos \phi, \sin \phi)$, for some $\phi \in[0,2 \pi)$, the first two components of the covector associated to an optimal trajectory and by $\theta(\cdot)$ the corresponding angle. Note that the evolution of $p_{3}$ is described by the equation

$$
\dot{p}_{3}(t)=\rho \sin (\theta(t)-\phi) .
$$

The two following results essentially show that the optimal trajectories behave almost as straight lines directed with the angle $\alpha$ defined above, on a large portion of the interval $[0, T]$ (this assertion will be made more precise in Theorem 3.13). Moreover, the curvature $\kappa(t)$ is uniformly bounded on the whole interval $[0, T]$.

Proposition 3.7: For every $\varepsilon>0$ there exists $\mathcal{T}_{\varepsilon}>0$ such that, for every optimal trajectory, one has $\mu\left(J_{\varepsilon}\right) \leq \mathcal{T}_{\varepsilon}$, where the set $J_{\varepsilon}$ is defined as

$$
J_{\varepsilon}=\{\tau \in[0, T]:|\alpha-\theta(\tau)| \geq \varepsilon\} .
$$

Proposition 3.8: There exists a constant $C_{\varphi, \psi}>0$ such that for any optimal trajectory $\|\kappa(t)\|_{L^{\infty}([0, T])} \leq C_{\varphi, \psi}$. Moreover, for every $\varepsilon>0$, there exists $\mathcal{T}_{\varepsilon}>0$ such that, for every optimal trajectory, one has $\mu\left(K_{\varepsilon}\right) \leq \mathcal{T}_{\varepsilon}$, where the set $K_{\varepsilon}$ is defined as

$$
K_{\varepsilon}=\{\tau \in[0, T]:|u(\tau)| \geq \varepsilon \text { or }|\kappa(\tau)| \geq \varepsilon\} .
$$

The next proposition states a uniform limit for the value of $\left(p_{1}, p_{2}\right)$ for final points far from the origin

Proposition 3.9: For every $\eta>0$ there exists $R_{\eta}>0$ such that for every optimal trajectory with $\left|\left(x_{1}, y_{1}\right)\right| \geq R_{\eta}$ one has $|\phi-\alpha| \leq \eta$ and $|\rho-1| \leq \eta$, where we recall that $\rho:=\left|\left(p_{1}, p_{2}\right)\right|$.

A limit asymptotic value for $\left(p_{3}, p_{4}\right)$ is not available at this stage, nevertheless the following result states the existence of a uniform bound on $\left(p_{3}, p_{4}\right)$ for far enough final points.

Proposition 3.10: There exist two positive constants $C_{\varphi, \psi}$ and $T_{\varphi, \psi}$ such that, for every optimal trajectory defined on $[0, T]$ with $T>T_{\varphi, \psi}$, one has $\left\|\left(p_{3}, p_{4}\right)\right\|_{L^{\infty}([0, T])} \leq$ $C_{\varphi, \psi}(1+\rho)$.

Remark 3.11: One immediately deduces from Proposition 3.10 and Proposition 3.9 that $p_{3}, p_{4}$ and $u$ are uniformly bounded for $T$ large enough over all optimal trajectories.

At first sight it seems reasonable to conjecture that the previous results can be improved in the following directions:

(a) extending the uniformity results to all optimal trajectories, i.e. independently of the final time $T$;

(b) as the terminal point $\left(x_{1}, y_{1}\right)$ goes to infinity, the corresponding optimal control $u(\cdot)$ tends to 0 .

However it is not difficult to show that the first conjecture is false and to find a counterexample to the second conjecture.
The following result essentially shows that, if the initial, final directions and the segment joining initial and final points are almost aligned, then the corresponding cost is not far from the distance among initial and final points.

Proposition 3.12: For every $C>0$ there exists $\delta>0$ and $R>0$ large enough such that the following holds. Let $W_{0}=\left(\bar{x}_{0}, \bar{y}_{0}, \theta_{0}, \kappa_{0}\right), W_{1}=\left(\bar{x}_{1}, \bar{y}_{1}, \theta_{1}, \kappa_{1}\right)$, and set $\left(\bar{x}_{1}-\right.$ $\left.\bar{x}_{0}, \bar{y}_{1}-\bar{y}_{0}\right)=\Gamma(\cos \bar{\theta}, \sin \bar{\theta})$ for some $\Gamma>0$ and $\bar{\theta} \in$ $[0,2 \pi]$. Then, if $\left|\theta_{i}-\bar{\theta}\right|<\delta,\left|\kappa_{i}\right|<\delta$ for $i=0,1$ and $\Gamma \geq R$, any optimal trajectory connecting $W_{0}$ to $W_{1}$ satisfies $J(u(\cdot), T) \leq\left|\left(\bar{x}_{1}-\bar{x}_{0}, \bar{y}_{1}-\bar{y}_{0}\right)\right|+C$.

Using the previous proposition and the other preliminary qualitative results we get the following theorem, which is the main result of this section.

Theorem 3.13: Let us associate to any extremal trajectory $X(\cdot)$ of (OCP) the function $Z(t)=\left(\theta(t), \kappa(t), p_{3}(t), p_{4}(t)\right)$. Given $\nu>0$ there exist $\tau_{\nu}>0$ and $\sigma_{\nu}>2 \tau_{\nu}$ such that, for every optimal trajectory with final time $T>\sigma_{\nu}$, one has $|Z(t)-(\alpha, 0,0,0)|<\nu$ for $t \in\left[\tau_{\nu}, T-\tau_{\nu}\right]$.

E. Numerical study of the asymptotic behavior of optimal trajectories and of the corresponding value of $p_{3}(0)$.

The main informations provided in the previous sections concerning (OCP) can be summarized as follows:

(i) Optimal trajectories starting from $X_{0}$ exist for every final data $X_{1}$,

(ii) If the spatial components $\left(x_{1}, y_{1}\right)$ of $X_{1}$ are far enough then optimal trajectories can be decomposed in three pieces corresponding to time intervals $[0, \bar{t}],[\bar{t}, T-\bar{t}]$, $[T-\bar{t}, T]$, where $\bar{t}$ can be thought independent of $X_{1}$ and the arc of the trajectory on $[\bar{t}, T-\bar{t}]$ is approximately a segment (the accuracy of the approximation depends on the size of $\bar{t}$ ),

(iii) Through the Pontryagin maximum principle we know that for any optimal trajectory there exist two scalars $(\rho, \phi)$ and two time-dependent functions $p_{3}(\cdot), p_{4}(\cdot)$ such that $Z(\cdot)=\left(\theta(\cdot), \kappa(\cdot), p_{3}(\cdot), p_{4}(\cdot)\right)$ satisfies the following equation

$$
\left\{\begin{array}{l}
\dot{\theta}=\kappa, \\
\dot{\kappa}=\left(\psi^{\prime}\right)^{-1}\left(p_{4}\right), \\
\dot{p}_{3}=\rho \sin (\theta-\phi), \\
\dot{p}_{4}=-p_{3}+\varphi(\kappa) .
\end{array}\right.
$$

Also, the relation

$$
\begin{gathered}
\hat{H}\left(\theta, \kappa, p_{3}, p_{4}\right):=\rho \cos (\theta-\phi)+p_{3} \kappa \\
+p_{4}\left(\psi^{\prime}\right)^{-1}\left(p_{4}\right)-1-\varphi(\kappa)-\psi\left(\left(\psi^{\prime}\right)^{-1}\left(p_{4}\right)\right)=0
\end{gathered}
$$

holds along any optimal trajectory. Moreover, if $\left(x_{1}, y_{1}\right)$ is large enough, $\rho$ is close to 1 and $\phi$ is close to the angle $\alpha$ such that $\left(x_{1}, x_{2}\right)=\left|\left(x_{1}, x_{2}\right)\right|(\cos \alpha, \sin \alpha)$.

The qualitative properties stressed above do not allow neither to understand the local behavior of optimal trajectories, in particular on the intervals $[0, \bar{t}],[T-\bar{t}, T]$ defined by the above Condition (ii), nor to find them numerically. However they detect some non-trivial asymptotic behaviour of the pair $(\rho, \phi)$ and of the initial data of (16), for large values of 
$\left(x_{1}, y_{1}\right)$. The analysis carried out in this section arises from the observation that, in order to understand the asymptotic shape of the optimal trajectories on $[0, \bar{t}],[T-\bar{t}, T]$, it would be enough to complete the informations about the initial data of (16). Indeed, as far as the initial datum of the equation is close to its asymptotic value (if it exists) and $(\rho, \phi)$ is close to $(1, \alpha)$, we know, from classical continuous dependence results for the solutions of differential equations, that the solution of (16) will in turn be close (on compact time intervals) to the solution of the asymptotic equation

$$
\left\{\begin{array}{l}
\dot{\theta}=\kappa, \\
\dot{\kappa}=\left(\psi^{\prime}\right)^{-1}\left(p_{4}\right), \\
\dot{p}_{3}=\sin (\theta-\alpha), \\
\dot{p}_{4}=-p_{3}+\varphi(\kappa) .
\end{array}\right.
$$

where we take as initial value the asymptotic value of the initial data for (16). In other words, a precise knowledge of the asymptotic behaviour of such initial data, for large $\left(x_{1}, y_{1}\right)$, would provide a tool to study numerically, through (18), the asymptotic shape of optimal trajectories on $[0, \bar{t}]$ (and, by symmetry, on $[T-\bar{t}, T]$ ).

Let us first notice that an asymptotic value for $p_{4}(0)$ is simply provided by evaluating (17) at time 0 , with the approximation $(\rho, \phi)=(1, \alpha)$. More precisely $p_{4}(0)$ coincides with a solution $z$ of the equation

$$
\cos (\pi / 2-\alpha)+z\left(\psi^{\prime}\right)^{-1}(z)-1-\psi\left(\left(\psi^{\prime}\right)^{-1}(z)\right)=0 .
$$

Since the map $\eta \mapsto \psi^{\prime}(\eta) \eta-\psi(\eta)=\int_{0}^{\eta}\left(\psi^{\prime}(\eta)-\psi^{\prime}(\mu)\right) d \mu$ is strictly increasing for $\eta \geq 0$, strictly decreasing for negative $\eta$ and goes to infinity for $|\eta|$ going to infinity, because of the strict convexity of $\psi$, and since $\psi \in \mathcal{C}^{1}$, we know that the previous equation has exactly one positive solution and one negative solution. Since $u(0)=\left(\psi^{\prime}\right)^{-1}\left(p_{4}(0)\right)$, this suggests the existence of two asymptotic behaviour for the trajectories of (16), each one corresponding to a candidate solution for (OCP). These two trajectories start from $X_{0}$ by turning on opposite directions.

To complete the informations about the asymptotic value of the initial data for (16) we need to investigate the possible values of $p_{3}(0)$. For this purpose we will develop below a numerical method based on the existence of a stable manifold for (18).

An equilibrium for (18) is given by $\left(\theta, \kappa, p_{3}, p_{4}\right)=$ $(\alpha, 0,0,0)$ and we know from Theorem 3.13 that, for solutions of (OCP) with $\left(x_{1}, y_{1}\right)$ far enough from the origin, the corresponding values of $\left(\theta(\cdot), \kappa(\cdot), p_{3}(\cdot), p_{4}(\cdot)\right)$ are close to this equilibrium on some interval $[\bar{t}, T-\bar{t}]$ for large $\bar{t}$ and $T$, which suggests some stability property of the equilibrium. It is actually easy to see that $Y_{e q}=(\alpha, 0,0,0)$ is not a stable equilibrium of the system. Indeed the linearized system around $Y_{e q}$ is

$$
\begin{gathered}
\dot{Y}=J\left(Y-Y_{e q}\right), \quad Y \in \mathbb{R}^{4} \\
J=\left(\begin{array}{cccc}
0 & 1 & 0 & 0 \\
0 & 0 & 0 & 1 / \psi^{\prime \prime}(0) \\
1 & 0 & 0 & 0 \\
0 & \phi^{\prime \prime}(0) & -1 & 0
\end{array}\right)
\end{gathered}
$$

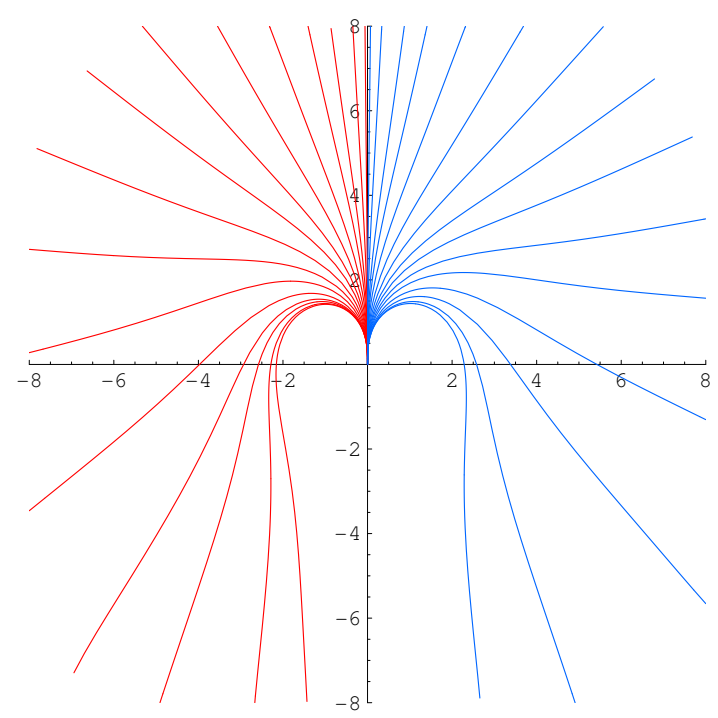

Fig. 3. Asymptotical behaviour of optimal trajectories with final point far from the origin.

where the matrix $J$ has exactly two eigenvalues $\lambda_{1}, \lambda_{2}$ with negative real part, corresponding to some eigenvectors $v_{1}, v_{2}$, while the other two eigenvalues $\mu_{1}, \mu_{2}$, with corresponding eigenvectors $w_{1}, w_{2}$, have positive real part. Therefore $Y_{e q}$ is a stable equilibrium for the linearized dynamics restricted to $Y_{e q}+V$, where $V$ is the two dimensional real subspace of $\mathbb{R}^{4}$ spanned by $v_{1}, v_{2}$ (notice that $v_{1}, v_{2}$ can be assumed either real or complex conjugate).

The classical stable manifold theorem (see for instance [7]) ensures the existence of a manifold $\mathcal{W}^{s}$ of dimension 2 , called stable manifold, which is tangent to $V$ and which contains all the trajectories converging to the equilibrium (exponentially fast). Note that, since the continuous function $\hat{H}$, with $(\rho, \phi)=(1, \alpha)$, is a first integral of the dynamics (18) and $\hat{H}\left(Y_{\text {eq }}\right)=0$ we have $\mathcal{W}^{s} \subset \hat{H}^{-1}(0)$.

On a small neighborhood of the equilibrium all the trajectories that are not contained in $\mathcal{W}^{s}$ diverge from it exponentially fast. Let us fix such a neighborhood $U$. From Theorem 3.13 we know that there exists $\bar{t}$ such that, if $Z(\cdot)$ is a trajectory of (16) associated to a solution of (OCP), then $Z(t) \in U$ for every $t \in[\bar{t}, T-\bar{t}]$, provided that $\left(x_{1}, y_{1}\right)$ is far enough from the origin. In particular if we consider a sequence of final points $X_{1}^{(n)}$ for (OCP) with spatial components $\left(x_{1}^{(n)}, y_{1}^{(n)}\right)=n(\cos \alpha, \sin \alpha)$ we deduce that, for the corresponding sequence of trajectories $Z^{(n)}(\cdot)$, the limit $\bar{Z}$ of $Z^{(n)}(\bar{t})$ exists (up to a subsequence) and is contained in $\mathcal{W}^{s}$. Continuous dependence results for the solutions of differential equations guarantee that the limit of $Z^{(n)}(0)$ coincides with $\bar{Z}(0)$, where $\bar{Z}(\cdot)$ is the solution of (18) such that $\bar{Z}(\bar{t})=\bar{Z}$. In particular it must be $\bar{Z}(0)=$ $\left(\pi / 2,0, \bar{p}_{3}, \bar{p}_{4}\right)$ where $\bar{p}_{4}$ satisfies (19).

The previous reasoning suggests a method to study numerically the possible values of $\bar{p}_{3}$ at time 0 . Indeed if $U$ is small enough then $\mathcal{W}^{s}$ is well approximated by the affine space $Y_{e q}+V$. Consequently one can numerically look for 


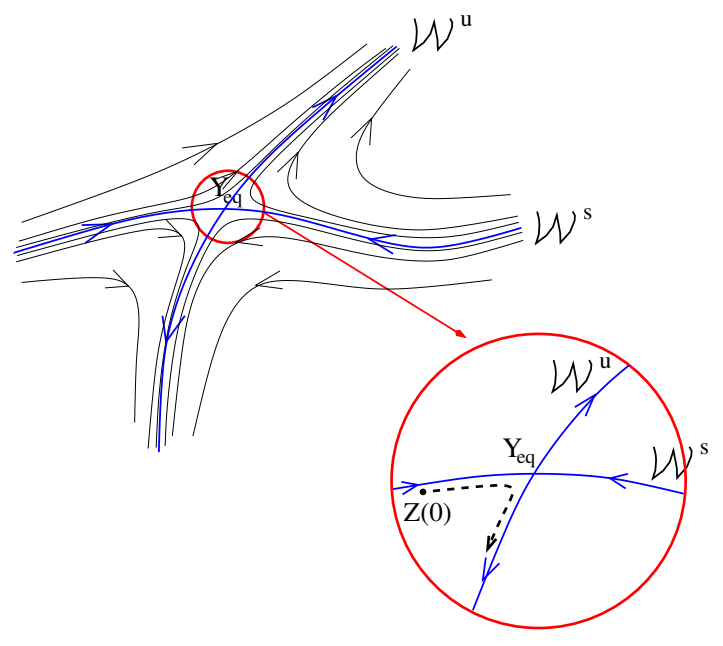

Fig. 4. Behaviour around the stable and unstable manifolds.

solutions of the asymptotic equation (18) with

$$
Z(\bar{t}) \in\left(Y_{e q}+V\right) \cap U
$$

and such that $\theta(0)=\pi / 2, \kappa(0)=0$. More precisely a simple numerical method can be specified as follows. Let us fix a closed curve $\gamma(s)=\varepsilon\left(\cos (s) \bar{v}_{1}+\sin (s) \bar{v}_{2}\right)$, where $\bar{v}_{1}, \bar{v}_{2}$ are real vectors spanning $V$ and $\varepsilon$ is a small constant (the precision of the method increases as $\varepsilon$ goes to zero). Since all the trajectories converging to the equilibrium must cross this curve (in the approximation $\mathcal{W}^{s} \simeq Y_{e q}+V$ ) we can recover them by following backwards in time the solutions of (18) starting at $Z(0)=Y_{\text {eq }}+\gamma(s)$ up to a time $\tilde{t}<0$ such that $\kappa(\tilde{t})=0$. The candidate approximate asymptotic trajectories we are looking for are then determined by the values of $s$ for which, for a reasonably not too large $\tilde{t}$ such that $\kappa(\tilde{t})=0$, we also have $\theta(\tilde{t})=\pi / 2$. The value $Z(0)$ is then a candidate value for the initial datum of a trajectory of (16) associated to a solution of (OCP), for large values of $\left(x_{1}, y_{1}\right)$. Moreover this simple method allows to approximate numerically the initial arc of such optimal trajectories (see Figure 3 which considers the case $\varphi \equiv 0, \psi(z)=z^{2}$ ).

An effective method to globally construct solutions of (OCP) for large values of $\left(x_{1}, y_{1}\right)$ is the following. Define a further closed curve $\hat{\gamma}(\hat{s})=\hat{\varepsilon}\left(\cos (\hat{s}) \bar{w}_{1}+\sin (\hat{s}) \bar{w}_{2}\right)$, where $\bar{w}_{1}, \bar{w}_{2}$ are real vectors generating the unstable subspace $W$ (defined similarly to $V$ ). Assume that $\hat{\varepsilon} \ll \varepsilon \ll 1$ and consider the solutions of (16) with $\phi=0$ and starting from $Z(0)=\gamma(s)+\hat{\gamma}(\hat{s})$, for suitable choices of $\rho, \varepsilon, \hat{\varepsilon}, s, \hat{s}$ such that $\hat{H}(Z(0))=0$. For a fixed small enough $\varepsilon>0$ and fixed $s \in[0,2 \pi]$ it turns out that the trajectory on intervals $\left[t_{1}, 0\right]$, with $t_{1}<0$ not too large, is subjected to small variations with respect to the choice of $\hat{\varepsilon} \ll \varepsilon, \hat{s} \in[0,2 \pi], \rho$ such that $\hat{H}(Z(0))=0$. In other words the trajectory approximately only depends on $\varepsilon, s$ on the interval $\left[t_{1}, 0\right]$. Similarly as before, the value $s$ and the time $t_{1}$ can be chosen in such a way that $\kappa\left(t_{1}\right)=0$ and, at the same time, $\theta\left(t_{1}\right)=\pi / 2-\phi$, for a prescribed value $\phi$.

On the other hand for positive time the components along the stable subspace $V$ decreases exponentially as far as the components along $W$ are small so that, after a certain time, the trajectory evolves close to the unstable manifold $\mathcal{W}^{u}$ (see Figure 4). The dynamics at this stage essentially depends on the initial choice of $\hat{\varepsilon}$ and $\hat{s}$, where the first parameter determines the time extent when the trajectory is confined inside $U$, while the second one essentially determines the final angle.

This method gives rise, up to a rotation of an angle $\phi$ and appropriate translations, to solutions of (OCP).

\section{PERSPECTIVES AND FUTURE WORK}

In this paper we have detected some important (asymptotic) properties of optimal trajectories that, in particular, show that the class of optimal control problems that we are considering is a reasonably good candidate for modeling the human locomotion. Also, these properties allow to simplify the computation of the optimal trajectories. Future work should follow at least the two following lines. First of all the locomotion trajectories determined experimentally should be compared with the optimal trajectories corresponding to the different costs considered here, in the spirit of [2], in order to determine the cost which best fits the experimental data. Our second objective is to derive an analytic justification to our choice of the family of costs to be minimized. The underlying idea is that the cost to be minimized shall possess some robustness properties with respect to small perturbation, that is that slightly different costs shall give origin to similar optimal trajectories; this similarity has a qualitative meaning (continuity of the trajectories with respect to the perturbation of the costs), and shall also be quantified (by means of sensitivity analysis). An important consequence of this fact would be that the study of complicated costs would become useless, since more simple and generic costs would determine similar optimal trajectories.

\section{REFERENCES}

[1] G. Arechavaleta, J-P. Laumond, H. Hicheur, and A. Berthoz. The nonholonomic nature of human locomotion: a modeling study. In IEEE / RAS-EMBS International Conference on Biomedical Robotics and Biomechatronics, Pisa (Italy), 2006.

[2] G. Arechavaleta, J-P. Laumond, H. Hicheur, and A. Berthoz. Optimizing principles underlying the shape of trajectories in goal oriented locomotion for humans. In IEEE / RAS International Conference on Humanoid Robots, Genoa (Italy), 2006.

[3] Gustavo Arechavaleta, Jean-Paul Laumond, Halim Hicheur, and Alain Berthoz. An optimality principle governing human walking. IEEE Transactions on Robotics, 24(1):5-14, 2008.

[4] Aram V. Arutyunov and Richard B. Vinter. A simple 'finite approximations' proofs of the Pontryagin maximum principle under reduced differentiability hypotheses. Set-Valued Anal., 12(1-2):5-24, 2004.

[5] T. Bayen, Y Chitour, F. Jean, and P. Mason. Asymptotic analysis of an optimal control problem connected to the human locomotion. Joint 48th IEEE Conference on Decision and Control and 28th Chinese Control Conference, Shanghai, 2009.

[6] T. Bayen, Y Chitour, F. Jean, and P. Mason. Analysis of an inverse optimal control problem connected to the human locomotion. Preprint LSS, December 2008.

[7] Anatole Katok and Boris Hasselblatt. Introduction to the modern theory of dynamical systems, volume 54 of Encyclopedia of Mathematics and its Applications. Cambridge University Press, Cambridge, 1995. With a supplementary chapter by Katok and Leonardo Mendoza.

[8] E. Trélat. Contrôle optimal. Mathématiques Concrètes. [Concrete Mathematics]. Vuibert, Paris, 2005. Théorie \& applications. [Theory and applications]. 